\title{
Disturbance-Observer-Based Adaptive Fuzzy Control for Islanded Distributed Energy Resource Systems
}

\author{
Chengshun Yang, ${ }^{1}$ Tao Hua $\left(D,{ }^{1}\right.$ Yuchen Dai ${ }^{D},{ }^{2}$ Guofu Liu, ${ }^{1}$ Xiaoning Huang, \\ and Dongdong Zhang ${ }^{1}$ \\ ${ }^{1}$ School of Electric Power Engineering, Nanjing Institute of Technology, Nanjing 211167, China \\ ${ }^{2}$ School of Automation, Wuhan University of Technology, Wuhan 430070, China \\ Correspondence should be addressed to Yuchen Dai; whutdyc@126.com
}

Received 3 October 2021; Revised 27 December 2021; Accepted 13 January 2022; Published 3 February 2022

Academic Editor: Weilin Yang

Copyright (C) 2022 Chengshun Yang et al. This is an open access article distributed under the Creative Commons Attribution License, which permits unrestricted use, distribution, and reproduction in any medium, provided the original work is properly cited.

\begin{abstract}
With the aim to improve the antidisturbance ability of the islanded distributed energy resource (DER) systems, a disturbanceobserver-based adaptive fuzzy sliding mode control (DAFSC) voltage controller is designed based on indirect vector control, which implements the voltage tracking and improves the self-regulation ability of the islanded DER systems. Firstly, the circuit diagram and the mathematical model of the DER system are presented. Then, the second-order sliding mode differentiator is designed to solve the problem of calculation expansion in the backstepping control method. To solve the influence of lumped disturbance on the system, a disturbance-observer is proposed to observe the unknown disturbance and compensate the controller feed-forward. Moreover, fuzzy control is proposed to reduce the dependence of the control effect on model accuracy. Finally, the stability of the controller is verified by Lyapunov stability theory, and the hardware in the loop results is given to verify that the control effect of the proposed DAFC controller has better dynamic performance compared with proportion-integral (PI) and the backstepping control strategy.
\end{abstract}

\section{Introduction}

With the gradual depletion of traditional energy and the increasing awareness of environmental protection, distributed energy resource (DER) is getting more and more attention because of its excellent economy and environmental protection, and has been developing rapidly and widely used in distribution networks [1-6]. On the face of the Earth, there are many remote areas with a lot of solar energy, such as mountains and islands; then, a DER system can be built to supply power to the microgrid by using photovoltaic panels, batteries, and power electronics $[7,8]$. There are many types of microgrid, which can be divided into island and grid connected according to whether it is interconnected with the public power grid; according to the current division, it can be divided into DC, AC, and AC $\backslash D C$ hybrid microgrid $[9,10]$. Because of regional reasons, some DER systems cannot interconnect with the enormous power grid $[11,12]$, and then the DER system is operating in the island mode.
Due to its small capacity and lack of mutual power transmission with the large power grid, the islanded DER system is challenging to maintain the output voltage and frequency stability when the load changes if the system lacks proper control. Thus, this will reduce the quality of the output voltage. As a result, it can cause damage to the power supply system and the electrical types of equipment $[13,14]$. Therefore, this paper mainly focuses on ensuring the output voltage stability of the islanded DER system. In addition, in the isolated island microgrid, due to its small capacity and poor system inertia, a more advanced controller must be selected in the selection of its controller to ensure the stable operation of the system [15].

Some scholars have done much research on the control strategies of islanded operation DER system. In reference [16], a fractional-order sliding mode control strategy is utilized in islanded DER system. The proposed control method in this article implements the tracking of the voltage under balanced load, unbalanced load, and 
nonlinear load, and the output voltage can be kept stable when the current is disorganized. However, the amplitude fluctuation of $d$-axis voltage is larger as the simulation result shows, and there is no control over the uncertainty part of the model. In reference [17], the droop control strategy is used to control the voltage and reactive power. Meanwhile, the distribution of active power in multiple microgrids is realized. However, the controller lacks consideration of model uncertainties as well, and the frequency recovery of the system is slow when the load is switched. Reference [18] presents an optimal secondary voltage control strategy based on droop and PQ control method for islanded multibus microgrid to tradeoff multibus voltage regulation and reactive power allocation between voltage modulation units, but the nonlinear load is not taken into account in the simulation; therefore, the simulation results only proved the control effect under linear load. Reference [19] utilizes a control strategy based on feed-forward signals in order to eliminate the impact of the load. However, the load is assumed to be balanced in the simulation, as in reference [18], the change in the nonlinear load is not considered. In the actual operation of the power system, there will be a large number of power electronics devices in the load. Thus, the load connected to the system is not limited to the linear load. In [20], a nonlinear model predictive control (NMPC) algorithm is utilized in the DER system to deal with the active power generation on the power generation side and realization of load forecasting. However, the output voltage is still fluctuating. Reference [21] proposes an algorithm for distributed generation (DG) units in the distribution network in a microgrid central controller to choose the best droop settings, and the security margins of islanded microgrid voltage have been improved in the article.

In recent years, nonlinear control technology has been developed rapidly [22]. Backstepping control, as one of the nonlinear control methods, was first proposed by American professor Kokotovic et al. in 1991 [23]. Backstepping control is based on the control method of reducing the order of nonlinear systems, and in this case, the high-order nonlinear system is decomposed into several subsystems which do not exceed the highest order of the system; then, the error volume, virtual control volume, and Lyapunov function of each subsystem are defined from the bottom layer to the whole system. Finally, the controller can be designed for the whole system by using the stability condition of the Lyapunov function to ensure the stability of the system [24,25]. But there is also a shortage of backstepping control methods, the differential swelling [26]. In order to solve this problem, the command filter is applied to the backstepping control method, which aims to solve the problem of the repeated derivation of the virtual controller in the backstepping controller, therefore reducing the amount of calculation in the system [27]. In the modeling of the DER system, the mathematical model is imprecise, and the parameters in the model are uncertain due to the dynamic characteristics of the system [28]. So, the adaptive method is used in this article to estimate the error of the model. Meanwhile, the projection operator is used to guarantee the boundedness of the estimate. An adaptive command-filtered backstepping (ACB) controller is used in reference [29] to solve the problem in induction motor drive systems. The results show the proposed controller has achieved a better control effect in the induction motor drive systems. In reference [30], a neural network is combined with the ACB controller to solve the problem in induction motor drive systems, and the better effect of the proposed control method has been proved compared with dynamic surface control by simulation results. In [31], the ACB controller combined with the observer is used in uncertain nonlinear systems, and two examples are provided to prove the validity of the controller. However, the boundedness of adaptive estimation is not considered in all references [29-31].

In this paper, a disturbance-observer-based adaptive fuzzy sliding mode control (DAFSC) method is proposed for the islanded DER system, and the control objective is to stabilize the system's output voltage without considering the current and the dynamic characteristics of the load. The main innovations of this paper are as follows:

(1) The unmodeled part of the system dynamics model is regarded as lumped disturbance, and a disturbanceobserver is designed to observe the lumped disturbance

(2) In order to reduce the dependence of control effect on model accuracy, fuzzy logic system (FLS) is used to approximate the nonlinear part of the dynamics model

(3) A second-order sliding mode differentiator (SOSMD) is designed to estimate the virtual control signal and avoid the direct derivation of the virtual controller

Firstly, according to the control objective of VSC, the voltage mathematical model of the inverter is established by using Kirchhoff's voltage and current law. Secondly, based on the established mathematical model, a second-order sliding mode differentiator is designed for the energy storage inverter to realize the tracking control of the system output voltage. Since the control effect of the model-based control method greatly depends on the model accuracy, in order to eliminate the influence of the unmodeled part of the system on the system, the unmodeled part is regarded as a centralized disturbance, and a disturbance-observer is designed for estimation, which further improves the effect of the controller. In addition, the controller integrates the integral synovial control. Therefore, the robustness and anti-interference ability of the controller have been greatly improved. Finally, in order to get rid of the influence of model accuracy on the control effect and improve the performance of the controller to the greatest extent, the problems of high-order nonlinearity and parameter uncertainty in the DER system are solved based on fuzzy logic system, so that the controller is more in line with engineering application.

The sections of this paper are structured as follows. Section 2 gives the circuit structure and dynamic model of the islanded DER system. The DAFSC controller is designed for the islanded DER system in Section 3, and the Lyapunov 
stability theorem proves the controller's stability. In Section 4 , the control effect of the proposed DAFSC controller has been demonstrated by comparing with proportion-integral (PI) and backstepping control strategy. Further, in Section 5, some conclusions are given.

\section{Problem Statement and Preliminaries}

2.1. Structure of the Islanded DER System. The structure of the islanded DER system is shown in Figure 1. From Figure 1 , we can obtain that the system consists of distributed energy resource $u_{\mathrm{d} c}$, DC link capacitor $C_{\mathrm{d} c}$, voltage source converter (VSC), control unit, three-phase load, and three-phase $L C$ filter, where $L$ and $C_{f}$ represent the value of capacitance and inductance, and $R$ represents the ohmic loss of the filter and VSC. The three-phase AC variables $u_{\mathrm{tabc}}$, $i_{\text {tabc }}, u_{\text {sabc }}$, and $i_{\text {sabc }}$ signify the AC side voltage and current of VSC and the DER system output voltage and current, respectively. In actual operation, the DC side of the system is connected to photovoltaic arrays in order to offer stable DC voltage. Thus, in order to ensure the stability of power supply, this paper uses a DC voltage source to assume the photovoltaic array offers a stable DC voltage.

The designed controller is based on vector control method, that is, three-phase AC variable which the controller needed is transformed to $\mathrm{d} q$-frame from $a b c$-frame, next, the signals in $\mathrm{d} q$-frame are delivered into the controller to calculate with the $\mathrm{d} q$-frame reference voltage $u_{\text {sdref }}, u_{\text {sqref }}$, then, the controller produces modulated signals $m_{\mathrm{d} q}$ in $\mathrm{d} q$-frame, and the signals in $\mathrm{d} q$-frame are transformed back to $a b c$-frame sent to the pulse width modulation (PWM) generator. In the PWM generator, the six-pulse signal is produced from comparing three-phase modulation wave and triangular wave with an amplitude of 1 , and the six-pulse signal is used to control the VSC.

In the design of the microgrid controller interconnected with the external power grid, we use the phaselocked loop (PLL) to lock the frequency and phase between the microgrid and the external grid. Considering that the islanded DER system is not interconnected with the external power grid, it is unable to provide a stable frequency reference by the system itself. Therefore, in this paper, the frequency and angular velocity are provided by a voltage-controlled oscillator (VCO) and then transmission to the $\mathrm{d} q$ converters [32].

2.2. Dynamic Model of the Islanded DER System. By using Kirchhoff's voltage and current law, the mathematical model of islanded DER system in Figure 1 has been established in this section. This paper studies the control of voltage source converter. The hybrid energy storage power supply is simplified to DC power supply. By stabilizing the DC voltage source and controlling the inverter in the system, the AC bus voltage of the islanded DER system is controlled $[16,33]$. The relationship between voltage and current in the system is described as follows [34]:

$$
\begin{aligned}
& C \frac{d \overrightarrow{u_{s}}}{\mathrm{~d} t}=\vec{i}-\overrightarrow{i_{s}}, \\
& L \frac{d \vec{i}}{d t}=\overrightarrow{u_{t}}-\overrightarrow{u_{s}}-R \vec{i},
\end{aligned}
$$

where $\vec{i}, \overrightarrow{i_{s}}, \overrightarrow{u_{t}}$, and $\overrightarrow{u_{s}}$ represent the space phasor of $i_{t a b c}$, $i_{s a b c}, u_{t a b c}$, and $u_{s a b c}$, respectively. To eliminate the variable $\vec{i}$ from (1) and (2), one obtains

$$
\frac{d^{2} \overrightarrow{u_{s}}}{d t^{2}}=-\frac{R}{L} \frac{d \overrightarrow{u_{s}}}{d t}-\frac{\overrightarrow{u_{s}}}{L C_{f}}-\frac{1}{C_{f}} \frac{d \overrightarrow{i_{s}}}{d t}-\frac{R \overrightarrow{i_{s}}}{L C_{f}}+\frac{\overrightarrow{u_{t}}}{L C_{f}} .
$$

The relationship between three-phase PWM modulating signals $\vec{m}$ and output voltage of the VSC $\overrightarrow{u_{t}}$ can be defined as

$$
\overrightarrow{u_{t}}=\frac{u_{d c}}{2} \vec{m}
$$

Thus, (3) can be described as

$$
\frac{d^{2} \overrightarrow{u_{s}}}{d t^{2}}=-\frac{R}{L} \frac{d \overrightarrow{u_{s}}}{d t}-\frac{\overrightarrow{u_{s}}}{L C_{f}}-\frac{1}{C_{f}} \frac{d \overrightarrow{i_{s}}}{d t}-\frac{R \overrightarrow{i_{s}}}{L C_{f}}+\frac{u_{d c} \vec{m}}{2 L C_{f}} .
$$

Transform each space phasor in (5) to the $d q$-frame, and (5) can be rewritten as

$$
\begin{aligned}
\frac{d\left[\left(u_{s d}+j u_{s q}\right) e^{j \varphi}\right]}{d t^{2}}= & -\frac{R}{L} \frac{d\left[\left(u_{s d}+j u_{s q}\right) e^{j \varphi}\right]}{d t}, \\
& -\frac{R\left(i_{s d}+j i_{s q}\right) e^{j \varphi}}{L C_{f}}+\frac{u_{d c}\left(m_{d}+j m_{q}\right) e^{j \varphi}}{2 L C_{f}}, \\
& -\frac{\left(u_{s d}+j u_{s q}\right) e^{j \varphi}}{L C_{f}}-\frac{1}{C_{f}} \frac{d\left[\left(u_{s d}+j u_{s q}\right) e^{j \varphi}\right]}{d t},
\end{aligned}
$$

where $\varphi$ represents the rotation angle between $\alpha$-axis and $d$-axis in park transformation provided by VCO. Split the left-hand side of the (6) to $d$-axis component and $q$-axis component, and (6) can be written as follows with considering the uncertainty of the model:

$$
\begin{aligned}
\frac{d^{2} u_{s d}}{\mathrm{~d} t^{2}}= & 2 \omega \frac{\mathrm{d} u_{s q}}{\mathrm{~d} t}-\frac{R}{L} \frac{\mathrm{d} u_{s d}}{\mathrm{~d} t}+\left(\omega^{2}-\frac{1}{L C_{f}}\right) u_{s d}+\frac{R \omega}{L} u_{s q} \\
& -\frac{1}{C_{f}} \frac{\mathrm{d} i_{s d}}{\mathrm{~d} t}+\frac{\omega}{C_{f}} i_{s q}-\frac{R}{L C_{f}} i_{s d}+\frac{u_{d c}}{2 L C_{f}} m_{d}+\delta_{d}, \\
\frac{\mathrm{d}^{2} u_{s q}}{\mathrm{~d} t^{2}}= & -2 \omega \frac{\mathrm{d} u_{s d}}{\mathrm{~d} t}-\frac{R}{L} \frac{\mathrm{d} u_{s q}}{\mathrm{~d} t}+\left(\omega^{2}-\frac{1}{L C_{f}}\right) u_{s q}-\frac{R \omega}{L} u_{s d} \\
& -\frac{1}{C_{f}} \frac{\mathrm{d} i_{s q}}{\mathrm{~d} t}-\frac{\omega}{C_{f}} i_{s d}-\frac{R}{L C_{f}} i_{s q}+\frac{u_{\mathrm{d} c}}{2 L C_{f}} m_{q}+\delta_{q},
\end{aligned}
$$

where $\delta_{d}$ and $\delta_{q}$ represent the lumped disturbances of $d$-axis and $q$-axis model, respectively. 


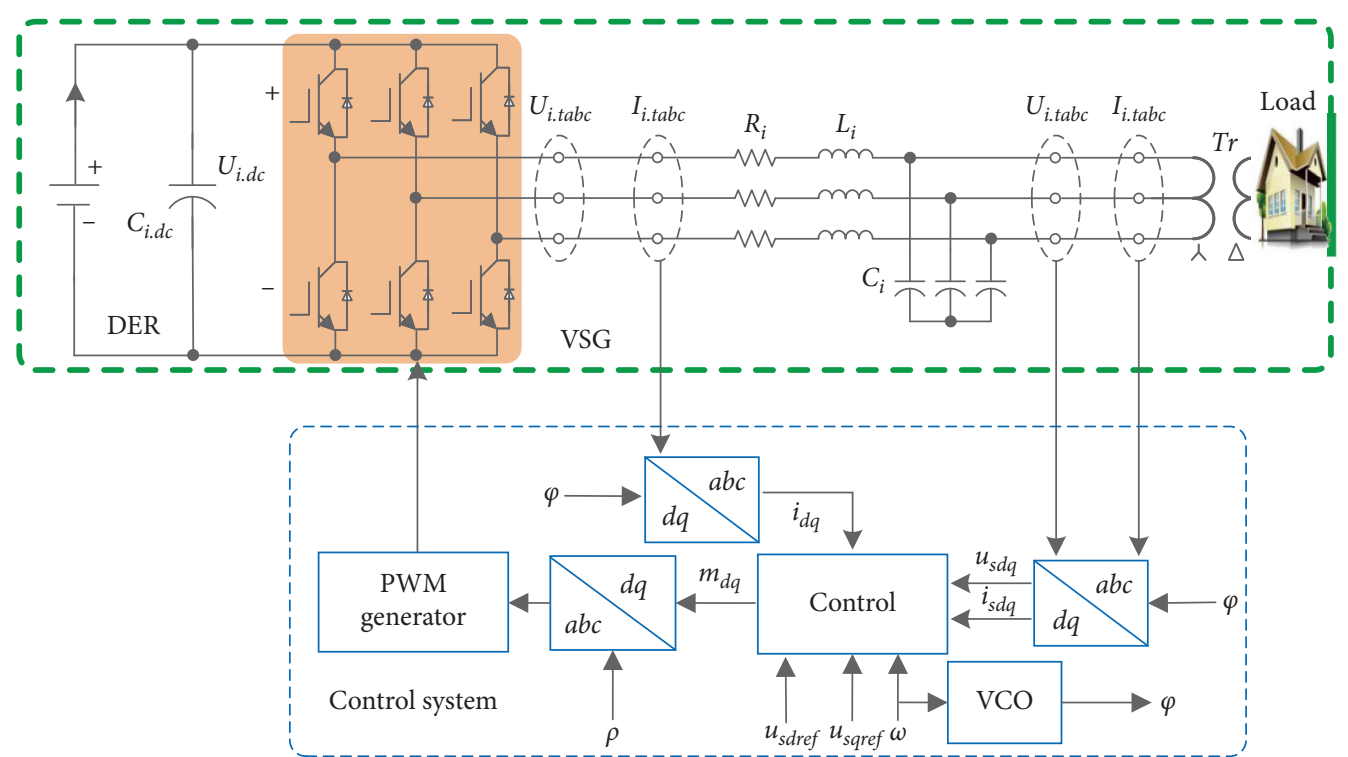

FIgURE 1: Structure of the islanded DER system.

\section{Design of the DAFSC Controller}

3.1. Design of the Disturbance-Observer. In this paper, the disturbance-observer is designed to observe the lumped disturbances of the system. The models (7) and (8) can be rewritten as

$$
\left\{\begin{array}{l}
\dot{x}_{1 . a}=x_{2 . a} \\
\dot{x}_{2 . a}=f_{a}(x)+g m_{a}+\delta_{a}
\end{array},\right.
$$

where $a=1,2, a=1$ indicates the model of $d$-axis, and $a=2$ indicates the model of $q$-axis. Moreover, $x_{1 . d}=u_{s d}$, $x_{1 . q}=u_{s q}, g=u_{d c} / 2 L C_{f}$, and

$$
\begin{aligned}
& f_{d}\left(u_{s d}, u_{s q}, i_{s d}, i_{s q}\right)=2 \omega \frac{\mathrm{d} u_{s q}}{\mathrm{~d} t}-\frac{R}{L} \frac{\mathrm{d} u_{s d}}{\mathrm{~d} t}+\left(\omega^{2}-\frac{1}{L C_{f}}\right) u_{s d}+\frac{R \omega}{L} u_{s q}-\frac{1}{C_{f}} \frac{d i_{s d}}{d t}+\frac{\omega}{C_{f}} i_{s q}-\frac{R}{L C_{f}} i_{s d}, \\
& f_{q}\left(u_{s d}, u_{s q}, i_{s d}, i_{s q}\right)=-2 \omega \frac{\mathrm{d} u_{s d}}{\mathrm{~d} t}-\frac{R}{L} \frac{\mathrm{d} u_{s q}}{\mathrm{~d} t}+\left(\omega^{2}-\frac{1}{L C_{f}}\right) u_{s q}-\frac{R \omega}{L} u_{s d}-\frac{1}{C_{f}} \frac{\mathrm{d} i_{s q}}{\mathrm{~d} t}-\frac{\omega}{C_{f}} i_{s d}-\frac{R}{L C_{f}} i_{s q} . \\
& \begin{array}{l}
\text { It is assumed that the lumped disturbance is differen- } \\
\text { ble and bounded. Taking the lumped disturbance as an } \\
\text { ditional state variable of the system, we have } x_{3 . a}=\delta_{a},
\end{array} \quad\left\{\begin{array}{l}
\dot{\tilde{x}}_{1 . a}=\tilde{x}_{2 . a}-\lambda_{1 . a} \operatorname{sig}^{(b+1) / 2}\left(\widetilde{x}_{1 . a}\right) \\
\dot{\tilde{x}}_{2 . a}=\tilde{x}_{3 . a}-\lambda_{2 . a} \operatorname{sig}^{(b+1) / 2}\left(\widetilde{x}_{1 . a}\right) \\
\dot{\tilde{x}}_{3 . a}=-\rho_{a}-\lambda_{3 . a} \operatorname{sig}^{b}\left(\tilde{x}_{1 . a}\right) .
\end{array}\right.
\end{aligned}
$$
tiable and bounded. Taking the lumped disturbance as an additional state variable of the system, we have $x_{3 . a}=\delta_{a}$, $\dot{\delta}_{a}=\rho_{a}$, and $\left|\rho_{a}\right| \leq \rho_{\text {d.a. }}$. Thus, (9) can be rewritten as

$$
\left\{\begin{array}{l}
\dot{x}_{1 . a}=x_{2 . a} \\
\dot{x}_{2 . a}=f_{a}(x)+g m_{a}+x_{3 . a} \\
\dot{x}_{3 . a}=\dot{\delta}_{a}=\rho_{a}(t)
\end{array}\right.
$$

Based on the model (11), the disturbance-observer can be designed as follows:

$$
\left\{\begin{array}{l}
\dot{\hat{x}}_{1 . a}=\hat{x}_{2 . a}-\lambda_{1 . a} \operatorname{sig}^{(b+1) / 2}\left(\tilde{x}_{1 . a}\right) \\
\dot{\hat{x}}_{2 . a}=f_{a}(x)+g m_{a}+\hat{x}_{3 . a}-\lambda_{2 . a} \operatorname{sig}^{(b+1) / 2}\left(\tilde{x}_{1 . a}\right) \\
\dot{\hat{x}}_{3 . a}=-\lambda_{3 . a} \operatorname{sig}^{(b+1) / 2}\left(\tilde{x}_{1 . a}\right)
\end{array}\right.
$$

where $\hat{x}_{i . a}$ is the estimated value of $x_{i . a}, \tilde{x}_{i . a}=\hat{x}_{i . a}-x_{i . a}$ are the observation errors, $\lambda_{i}>0$ is the observer gain, $0<b<1$, and $\operatorname{sig}^{\gamma}(x)=\operatorname{sgn}(x)|x|^{\gamma}$.

Then, the observer error system can be calculated as
Theorem 1 (see [35]). Assuming that the lumped disturbance $\delta_{a}$ is differentiable and bounded, considering the disturbance-observer (12), there exist the observer gains $\lambda_{1 . a}>0$, $\lambda_{2 . a}>0, \lambda_{3 . a}>0$, and $0<b<1$, such that the observation error can be converged, that is, $\left|x_{3 . a}\right| \leq \ell_{a}$.

3.2. Design of the Virtual Controller. Definition of $d$-axis tracking errors is as follows:

$$
\begin{aligned}
& e_{1}=u_{s \mathrm{~d}}-u_{\text {sdref }}, \\
& e_{2}=\dot{u}_{s d}-y_{d}^{c}=y_{d}-y_{d}^{c},
\end{aligned}
$$

where $y_{d}^{c}$ is the output signal of the virtual controller pass through the SOSMD. Take the derivative of two tracking errors in (14) and (15): 


$$
\begin{aligned}
& \dot{e}_{1}=\dot{u}_{s \mathrm{~d}}-\dot{u}_{\text {sdref }}, \\
& \dot{e}_{2}=\dot{y}_{d}-\dot{y}_{d}^{c} .
\end{aligned}
$$

In order to stabilize (15), the Lyapunov function is designed as follows:

$$
V_{d}=\frac{1}{2} e_{1}^{2}
$$

And, the derivative of the Lyapunov function is calculated as

$$
\begin{aligned}
\dot{V}_{d} & =e_{1} \dot{e}_{1}=e_{1}\left(y_{d}-\dot{u}_{\text {sdref }}\right) \\
& =-k_{1} e_{1}^{2}+e_{1}\left(k_{1} e_{1}+y_{d}-\dot{u}_{\text {sdref }}\right),
\end{aligned}
$$

where $k_{1}$ is a constant greater than zero. To make $\dot{V}_{d} \leq 0$, the virtual controller $y_{d}^{d}$ is chosen as

$$
y_{d}^{d}=-k_{1} e_{1}+\dot{u}_{\text {sdref }} \text {. }
$$

When a back stepping control strategy is designed in a high-order nonlinear system, due to repeated guidance to the virtual controller, the amount of calculation is expanded, and the controller's response speed and control effect are affected. As a result, in view of this problem, the SOSMD is designed to estimate the virtual control signal, which is designed as

$$
\left\{\begin{array}{l}
\dot{\eta}_{1 . a}=\tau_{1 . a} \\
\tau_{1 . a}=-\beta_{1}\left|\eta_{1 . a}-y_{a}^{d}\right|^{1 / 2} \operatorname{sign}\left(\eta_{1 . a}-y_{a}^{d}\right)+\eta_{2 . a} \\
\dot{\eta}_{2 . a}=-\beta_{2} \operatorname{sign}\left(\eta_{2 . a}-\tau_{1 . a}\right),
\end{array}\right.
$$

where $\beta_{1}$ and $\beta_{2}$ are positive constants and $\eta_{1 . a}$ and $\tau_{1 . a}$ are the estimated values of $y_{a}^{c}$ and $\dot{y}_{a}^{c}$, respectively.

However, a filter error will be generated during the operation of the SOSMD. To compensate for the filtering error, the tracking error $e_{1}$ should be redefined as

$$
\bar{e}_{1}=e_{1}-\varepsilon_{1},
$$

where the $\varepsilon_{1}$ is the compensation signal of filter error defined as

$$
\dot{\varepsilon}_{1}=-k_{1} \varepsilon_{1}+\left(y_{d}^{c}-y_{d}^{d}\right)
$$

Definition of $q$-axis tracking errors is as follows:

$$
\begin{aligned}
& e_{3}=u_{s q}-u_{\text {sqref }}, \\
& e_{4}=\dot{u}_{s q}-y_{q}^{c}=y_{q}-y_{q}^{c},
\end{aligned}
$$

The $y_{q}^{c}$ is the output of the $q$-axis SOSMD in (24). The derivative of (23) and (24) is calculated as

$$
\begin{aligned}
& \dot{e}_{3}=\dot{u}_{s q}-\dot{u}_{\text {sqref }}, \\
& \dot{e}_{4}=\dot{u}_{q}-\dot{u}_{q}^{c} .
\end{aligned}
$$

To stabilize (25), the Lyapunov function is defined as

$$
V_{q}=\frac{1}{2} e_{3}^{2}
$$

The derivative of (26) is calculated as

$$
\begin{aligned}
\dot{V}_{q} & =e_{3} \dot{e}_{3}=e_{3}\left(y_{q}-\dot{u}_{\text {sqref }}\right) \\
& =-k_{3} e_{3}^{2}+e_{3}\left(k_{3} e_{3}+y_{q}-\dot{u}_{\text {sqref }}\right),
\end{aligned}
$$

where $k_{3}$ is a constant greater than zero. Also, for the sake of making $\dot{V}_{q} \leq 0$, the virtual controller $y_{q}^{d}$ is chosen as

$$
y_{q}^{d}=-k_{3} e_{3}+\dot{u}_{\text {sqref }} .
$$

Same as the $d$-axis design process, the virtual controller $y_{q}^{d}$ is sent to the SOSMD to get signal $y_{q}^{c}$ and $\dot{y}_{q}^{c}$. Redefine the tracking error $e_{3}$ as

$$
\bar{e}_{3}=e_{3}-\varepsilon_{2},
$$

where the compensation signal of filter error $\varepsilon_{2}$ is designed as

$$
\dot{\varepsilon}_{2}=-k_{3} \varepsilon_{2}+\left(y_{q}^{c}-y_{q}^{d}\right) \text {. }
$$

According to (22), the derivative of error $\bar{e}_{1}$ can be calculated as

$$
\begin{aligned}
\dot{\bar{e}}_{1} & =\dot{e}_{1}-\dot{\varepsilon}_{1} \\
& =\dot{u}_{s d}-y_{d}^{c}+k_{1} \varepsilon_{1}-k_{1} e_{1}-\dot{u}_{\text {sdref }}+\dot{u}_{\text {sdref }} \\
& =-k_{1} \bar{e}_{1}+e_{2} .
\end{aligned}
$$

Similarly, on the basis of (30), the derivative of $\bar{e}_{3}$ can be calculated as

$$
\begin{aligned}
\dot{\bar{e}}_{3} & =\dot{e}_{3}-\dot{\varepsilon}_{2} \dot{u}_{s q}-y_{q}^{c}+k_{3} \varepsilon_{2}-k_{3} e_{3}-\dot{u}_{\text {sqref }}+\dot{u}_{\text {sqref }} \\
& =-k_{3} \bar{e}_{3}+e_{4} .
\end{aligned}
$$

The derivative of errors $e_{2}$ and $e_{4}$ is calculated as follows: $\dot{e}_{2}=\dot{y}_{d}-\dot{y}_{d}^{c}=f_{d}\left(u_{s d}, u_{s q}, i_{s d}, i_{s q}\right)+g m_{d}+\delta_{d}-\dot{y}_{d}^{c}$,

$$
\dot{e}_{4}=\dot{y}_{q}-\dot{y}_{q}^{c}=f_{q}\left(u_{s d}, u_{s q}, i_{s d}, i_{s q}\right)+g m_{q}+\delta_{q}-\dot{y}_{q}^{c} .
$$

According to the following fuzzy rules to construct the fuzzy system [36-38],

$$
\text { IF } x_{1} \quad \text { is } A_{1}^{l_{1}} \text { and } \cdots \text { and } x_{n} \text { is } A_{n}^{l_{n}} \text {, THEN } u_{d} \text { is } S^{l_{1} \cdots l_{n}} \text {, }
$$

where $l_{1}=1,2 \ldots, m_{i}, i=1,2, \ldots, n$.

Using product reasoning machine, single-value fuzzer and center-average fuzzer design the following fuzzy controllers:

$$
f(x \mid \theta)=\sum_{i=1}^{N} \Phi_{i} \frac{\prod_{j=1}^{n} \mu_{j}^{i}\left(x_{j}\right)}{\sum_{i=1}^{N} \prod_{j=1}^{n} \mu_{j}^{i}\left(x_{j}\right)=\Phi^{T} Y(x),}
$$

where $\Phi=\left[\phi_{1}, \phi_{2}, \ldots, \phi_{n}\right]^{T}$ is the unknown idea parameter vector; $\mu_{j}^{i}\left(x_{j}\right)$ is the memory of fuzzy system; and $\Upsilon(x)=$ $\left[\gamma_{1}(x), \gamma_{2}(x), \ldots, \gamma_{n}(x)\right]^{T}$ is the fuzzy basis function vector, in which

$$
Y(x)=\frac{\prod_{i=1}^{n} \mu_{j}^{i}\left(x_{j}\right)}{\sum_{i=1}^{N} \prod_{j=1}^{n} \mu_{j}^{i}\left(x_{j}\right)}
$$

Lemma 1 (see [39]). A continuous function $f(x)$ is delimited on a compact set $\Omega$. For any positive scalar 1 , there exists a FLS $\Phi^{T} \Upsilon(x)$ that meets the following relationship: 


$$
\sup _{x \in \Omega}\left|f(x)-\Phi^{T} \Upsilon(x)\right| \leq \iota
$$

According to the approximation principle of fuzzy system, if $g(x)$ is defined as the function set of the system, for any constant $\varepsilon>0$ there exists a fuzzy system satisfying $|g(x)-f(x)| \leq \varepsilon$.

Considering the nonlinear parts of the dynamics model is approximated by FLS, which is expressed as

$$
\begin{aligned}
& f_{d}=\Phi_{d}^{T} \Upsilon_{d}(x)+o_{d}, \\
& f_{q}=\Phi_{q}^{T} \Upsilon_{q}(x)+o_{q} .
\end{aligned}
$$

where $\left|o_{a}\right| \leq \iota_{a}$, and we can get the unequal relation as

$$
\begin{aligned}
& e_{2} f_{d} \leq \frac{\left\|\Phi_{d}\right\|^{2}}{2 h_{d}^{2}} \Upsilon_{d}^{T} \Upsilon_{d} e_{2}^{2}+\frac{1}{2} h_{d}^{2}+\frac{1}{2} l_{d}^{2}+\frac{1}{2} e_{2}^{2} . \\
& e_{4} f_{q} \leq \frac{\left\|\Phi_{q}\right\|^{2}}{2 h_{q}^{2}} \Upsilon_{q}^{T} \Upsilon_{q} e_{4}^{2}+\frac{1}{2} h_{q}^{2}+\frac{1}{2} l_{q}^{2}+\frac{1}{2} e_{4}^{2} .
\end{aligned}
$$

In order to stabilize the whole system, the Lyapunov function is defined again

$$
V_{t}=\frac{1}{2}\left(\bar{e}_{1}^{2}+e_{2}^{2}+\bar{e}_{3}^{2}+e_{4}^{2}+\frac{\widetilde{\Xi}^{2}}{\Gamma}\right),
$$

where $\Gamma$ is the positive scalar, $\Xi=\max \left\{\left\|\Phi_{d}\right\|^{2},\left\|\Phi_{q}\right\|^{2}\right\}$, $\widehat{\Xi}$ is the estimate value of $\Xi, \widetilde{\Xi}$ is the estimation error satisfying $\widetilde{\Xi}=\widehat{\Xi}-\Xi$, and $h_{a}$ is the positive scalar.

The derivative of Lyapunov function (42) is calculated as

$$
\dot{V}_{t}=\bar{e}_{1} \dot{\bar{e}}_{1}+e_{2} \dot{e}_{2}+\bar{e}_{3} \dot{\bar{e}}_{3}+e_{4} \dot{e}_{4}+\frac{\widetilde{\Xi} \dot{\vec{\Xi}}}{\Gamma} .
$$

Substituting (32)-(35), (40), and (41) into (43), one obtains

$$
\begin{aligned}
\dot{V}_{t} \leq & -k_{1} \bar{e}_{1}^{2}-k_{2} e_{2}^{2}-k_{3} \bar{e}_{3}^{2}-k_{4} e_{4}^{2}+e_{2}\left(\bar{e}_{1}+\frac{\widehat{\Xi}}{2 h_{d}^{2}} \Upsilon_{d}^{T} \Upsilon_{d} e_{2}+g m_{d}+\delta_{d}-\dot{y}_{d}^{c}+k_{2} e_{2}\right) \\
& +e_{4}\left(\bar{e}_{3}+\frac{\widehat{\Xi}}{2 h_{q}^{2}} \Upsilon_{q}^{T} \Upsilon_{q} e_{4}+g m_{q}+\delta_{q}-\dot{y}_{q}^{c}+k_{4} e_{4}\right)+\frac{\widetilde{\Xi}}{\Gamma}\left(\dot{\widehat{\Xi}}-\left(\frac{\Gamma}{2 h_{d}^{2}} \Upsilon_{d}^{2} \Upsilon_{d} e_{2}^{2}+\frac{\Gamma}{2 h_{q}^{2}} \Upsilon_{q}^{2} \Upsilon_{q} e_{4}^{2}\right)\right) \\
& +\frac{1}{2} h_{d}^{2}+\frac{1}{2} l_{d}^{2}+\frac{1}{2} e_{2}^{2}+\frac{1}{2} h_{q}^{2}+\frac{1}{2} l_{q}^{2}+\frac{1}{2} e_{4}^{2},
\end{aligned}
$$

where $k_{2}$ and $k_{4}$ are positive scalar.

Based on (44), the $d$-axis and the $q$-axis controllers are designed as

$$
\begin{aligned}
& m_{d}=\frac{1}{g}\left(-k_{2} e_{2}-\bar{e}_{1}-\frac{\widehat{\Xi}}{2 h_{d}^{2}} \Upsilon_{d}^{T} \Upsilon_{d} e_{d}-\widehat{\delta}_{d}+\dot{y}_{d}^{c}\right), \\
& m_{q}=\frac{1}{g}\left(-k_{4} e_{4}-\bar{e}_{3}-\frac{\widehat{\Xi}}{2 h_{q}^{2}} \Upsilon_{q}^{T} \Upsilon_{q} e_{q}-\widehat{\delta}_{q}+\dot{y}_{q}^{c}\right) .
\end{aligned}
$$

The parameter updating law $\dot{\widehat{\Xi}}$ is constructed as

$$
\dot{\hat{\Xi}}=\frac{\Gamma}{2 h_{d}^{2}} \Upsilon_{d}^{2} \Upsilon_{d} e_{d}^{2}+\frac{\Gamma}{2 h_{q}^{2}} \Upsilon_{q}^{2} \Upsilon_{q} e_{q}^{2}-\sigma \widehat{\Xi} .
$$

Substituting (45), (46), and (47) into (44), the derivative of Lyapunov function $\dot{V}_{t}$ can be expressed as

$$
\begin{aligned}
\dot{V}_{t} \leq & -k_{1} \bar{e}_{1}^{2}-k_{2} e_{2}^{2}-k_{3} \bar{e}_{3}^{2}-k_{4} e_{4}^{2}-\frac{\sigma \widetilde{\Xi} \widehat{\Xi}}{\Gamma}+e_{2} \ell_{d} \\
& +e_{4} \ell_{q}+\frac{1}{2} h_{d}^{2}+\frac{1}{2} l_{d}^{2}+\frac{1}{2} e_{2}^{2}+\frac{1}{2} h_{q}^{2}+\frac{1}{2} l_{q}^{2}+\frac{1}{2} e_{q}^{2} .
\end{aligned}
$$

Lemma 2 (see [40]). Young's inequality: for any non-negative real number $x, y$, the following inequality holds:

$$
x y \leq \frac{x^{p}}{p}+\frac{y^{q}}{q},
$$

where $p>1$ and $1 / p+1 / q=1$. If and only if $x^{p}=y^{q}$, the equal sign holds.

Based on Young's inequality, one obtains

$$
\begin{aligned}
& e_{2} \ell_{d} \leq \frac{1}{2} e_{2}^{2}+\frac{1}{2} \ell_{d}^{2}, \\
& e_{4} \ell_{q} \leq \frac{1}{2} e_{4}^{2}+\frac{1}{2} \ell_{q}^{2}, \\
& -\widetilde{\Xi} \widehat{\Xi} \leq-\frac{\widetilde{\Xi}^{2}}{2}+\frac{\Xi^{2}}{2} .
\end{aligned}
$$

Then, (48) can be rewritten as

$$
\begin{aligned}
\dot{V}_{t} & \leq-k_{1} \bar{e}_{1}^{2}-\left(k_{2}-1\right) e_{2}^{2}-k_{3} \bar{e}_{3}^{2}-\left(k_{4}-1\right) e_{4}^{2}-\frac{\sigma \widetilde{\Xi}^{2}}{2 \Gamma} \\
& +\frac{1}{2} h_{d}^{2}+\frac{1}{2} l_{d}^{2}+\frac{1}{2} h_{q}^{2}+\frac{1}{2} l_{q}^{2}+\frac{1}{2} \ell_{d}^{2}+\frac{1}{2} \ell_{q}^{2}+\frac{\sigma \Xi^{2}}{2 \Gamma} \\
& \leq-\mathscr{A} V_{t}+\mathscr{B} .
\end{aligned}
$$

where $\mathscr{A}=\min \left\{2 k_{1}, 2\left(k_{2}-1\right), 2 k_{3}, 2\left(k_{4}-1\right), \sigma\right\}$ and $\mathscr{B}=+$ $1 / 2 h_{d}^{2}+1 / 2 \iota_{d}^{2}+1 / 2 h_{q}^{2}+1 / 2 \iota_{q}^{2}+1 / 2 \ell_{d}^{2}+1 / 2 \ell_{q}^{2}+\sigma \Xi^{2} / 2 \Gamma$. As a 
TABLE 1: The basic parameters of the islanded DER system.

\begin{tabular}{lcc}
\hline Parameters & Value & Description \\
\hline$L$ & $300 \mu \mathrm{H}$ & Filter inductor \\
$C_{f}$ & $500 \mu \mathrm{F}$ & Filter capacitance \\
$R$ & $3 \mathrm{~m} \Omega$ & Filter resistance \\
$u_{d c}$ & $1800 \mathrm{~V}$ & DC link voltage \\
$\omega$ & $100 \pi \mathrm{rad} / \mathrm{s}$ & Nominal angular frequency \\
\hline
\end{tabular}

result, we can get the conclusion that the whole system is asymptotically stable at the origin.

\section{Results and Analysis}

In order to verify the effectiveness of the designed controller, according to the islanded DER model shown in Figure 1, a simulation model is built by using MATLAB/Simulink software in this section. The parameters in the DER system are listed in Table 1. For the sake of testing the dynamic characteristics of the controller, different types of load are used during the simulation process, including balanced load, unbalanced load, and nonlinear load. Table 2 shows the parameters of different types of loads.

It is important to adjust the controller and adaptive parameters to make sure the controller has a good effect. The adaptive parameters $r_{1}$ and $r_{2}$ are set to zero at first; owing to the control process in $d q$-frame, there is no coupling between the $d$-axis and $q$-axis; therefore, the $d$-axis controller parameters $k_{1}, k_{2}$ and $q$-axis controller parameters $k_{3}, k_{4}$ can be adjusted separately. Then, adjust the adaptive parameters to make the system achieve a satisfactory state. The controller parameters and adaptive parameters are listed in Table 3.

Remark 1. The parameter selection steps of the DAFSC controller are as follows: firstly, determine the membership function and set the value of adaptive rate $\Gamma$ from small to large so that the adaptive fuzzy logic system can achieve the goal of approaching the nonlinear function in the inverter model of the energy storage system. The adaptive learning rate $\sigma$ is selected between $0.001^{\sim}$. A too high learning rate will cause the instability of FLS, and too small will slow down the adaptive convergence speed. After that, the inner loop parameters $k_{2}, k_{4}$ are adjusted. Then, the outer loop parameters $k_{1}, k_{3}$ are adjusted to realize the accurate tracking control of the output voltage of DER. Finally, the damping and bandwidth of the instruction filter and the gain coefficient of the disturbance-observer are adjusted. The performance of the command filter depends on the damping and bandwidth. During the debugging of the command filter, it should be observed whether the output signal can approach the input signal. If so, the damping and bandwidth values at this time are appropriate. To adjust the gain coefficient of the disturbance-observer, it is necessary to observe whether the observed value can approach the actual disturbance value. If so, the gain coefficient at this time is appropriate. The value of the gain coefficient should not be too large. Otherwise, it will increase the influence of the estimated peak.
The whole simulation process lasts $2.5 \mathrm{~s}$. At the start of the simulation, the balanced load is switched on, the reference voltage of $d$-axis $u_{s d r}$ ef starts to rise straight from $0 \mathrm{~V}$ and reaches $480 \mathrm{~V}$ when $t=0.1 \mathrm{~s}$, at $t=2 \mathrm{~s}, u_{s d r \text { ef }}$ is changed stepwise to $530 \mathrm{~V}$ from $480 \mathrm{~V}$, and the $q$-axis reference voltage $u_{\text {sqref }}$ is maintained at $0 \mathrm{~V}$ in the whole simulation. On the load side, the load of the system is switched into unbalanced loads and nonlinear loads at $t=0.9 \mathrm{~s}$ and $t=1.8 \mathrm{~s}$, respectively.

Figures 2 and 3 show the voltage tracking effect and tracking error surfaces under proportion-integral (PI) control, respectively. As we can see from Figures 2 and3, when the load is switched from three-phase balanced load to three-phase unbalanced load at $t=0.9 \mathrm{~s}$, the $d$-axis voltage tracking error rises from $2 \mathrm{~V}$ to about $5 \mathrm{~V}$, and $q$-axis voltage tracking error rises from $4 \mathrm{~V}$ to about $6 \mathrm{~V}$; when the load is switched from three-phase unbalance load to nonlinear load at $t=1.8 \mathrm{~s}$, the voltage tracking error is further expanded.

Figures 4 and 5 show the voltage tracking effect and tracking error surfaces under backstepping control strategy, respectively. By comparison with Figures 4 and 5 under PI control strategy, it can be seen that the voltage keeps steady tracking when the load changes. However, the voltage tremor of $d$-axis and $q$-axis is still too large. Moreover, when $u_{s d r}$ ef is changed stepwise to $510 \mathrm{~V}$ from $450 \mathrm{~V}$ at $t=2 \mathrm{~s}$, the output voltage of the $d$-axis produces an overshoot of about $30 \mathrm{~V}$ when the step signal occurs.

Figures 6 and 7 show the voltage tracking effect and tracking error surfaces under the DAFSC strategy, respectively. When the load type is switched in the system at $t=0.9 \mathrm{~s}$ and $t=1.8 \mathrm{~s}$, the tracking error does not produce fluctuations by using the DAFSC controller. In addition, when a step signal is generated by $d$-axis reference voltage at $t=2 \mathrm{~s}$, the $d$-axis output voltage resumes tracking quickly and smoothly, and no overshoot and distortion appear. Furthermore, the tracking error of $d$-axis and $q$-axis is maintained within $1 \mathrm{~V}$ in the whole process of simulation under the DAFSC strategy. Therefore, through the simulation of the three kinds of control methods, it is proved that the DAFSC controller designed in this paper has better performance and robustness than traditional PI and backstepping control.

Figures 8 and 9 show the three-phase voltage and current waveforms at the time of load switching under the DAFSC strategy. It can be seen that the three-phase current is distorted when the load is switched to an unbalanced load or nonlinear load. Nonetheless, the voltage remained stable. Figure 10 contrasts the input signal $y_{d}$ and output signal $y_{d}^{c}$ of the SOSMD. The input and output signals are almost identical. 
Table 2: Different types of load.

\begin{tabular}{lcc}
\hline Load type & Load description & $\begin{array}{c}\text { Transformer } \\
\text { ratio }\end{array}$ \\
\hline Balanced load & Three star-connected $R L$ series load with $R=190 \Omega$ and $L=327 \mu H$. \\
Unbalanced load & $R L$ series Load with $R=150 \Omega$ and $L=1.24 H$ in phase-a, $R L$ series load with $R=140 \Omega$ and \\
$L=229 \mathrm{mH}$ in phase-b, phase-c is open. & $5 \mathrm{kV}_{\text {rms }} / 3.3 \mathrm{kV}$ rms \\
Nonlinear load & Uncontrollable three-phase bridge rectifier circuit, series $R L$ circuits with $R=630 \Omega$ and \\
$L=1.67 \mathrm{H}$ in DC side.
\end{tabular}

TABle 3: The basic parameters of the islanded DER system.

\begin{tabular}{lcc}
\hline Parameters & Value & Description \\
\hline$k_{1}, k_{2}$ & 10000,12000 & Gains of $d$-axis controller \\
$k_{3}, k_{4}$ & 9000,13000 & Gains of $q$-axis controller \\
$\Gamma, \sigma$ & $10,0.1$ & Gains of adaptive \\
$\lambda_{1} \cdot a$ & $3 \times 10000$ & Gains of disturbance-observer \\
$\lambda_{2} \cdot a$ & $3 \times 10000^{2}$ & Gains of disturbance-observer \\
$\lambda_{3} \cdot a$ & $10000^{2}$ & Gains of disturbance-observer \\
\hline
\end{tabular}

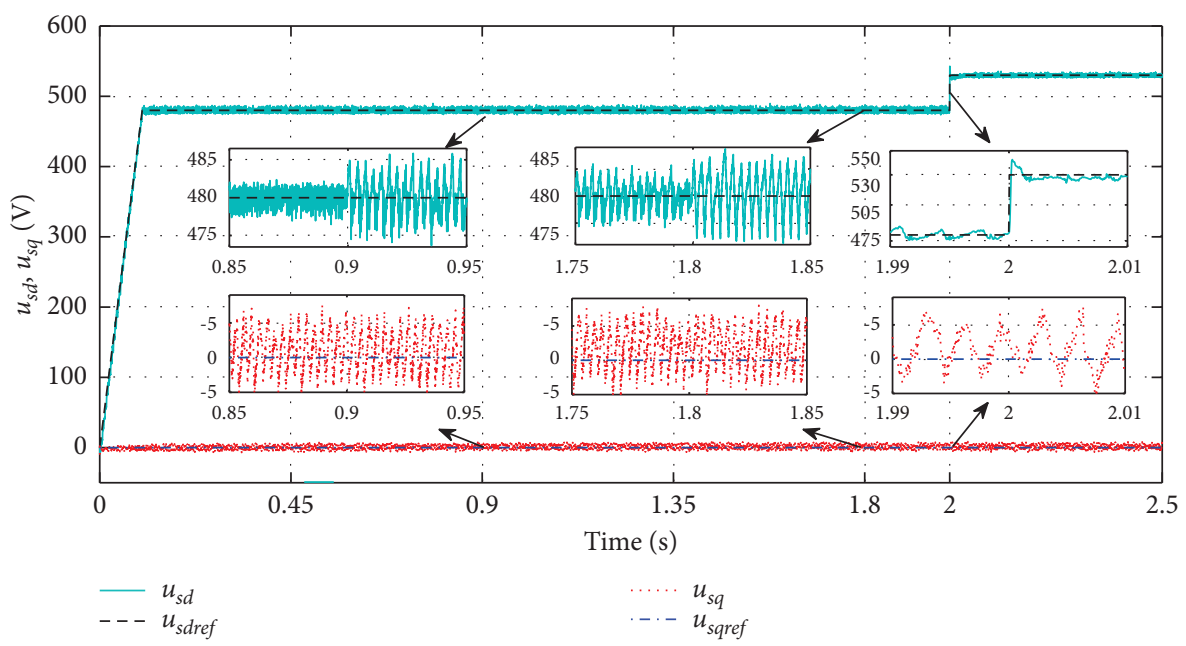

FIgURE 2: Voltage tracking effect under PI control strategy.

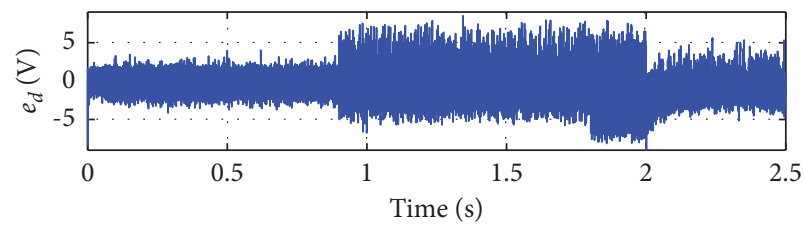

(a)

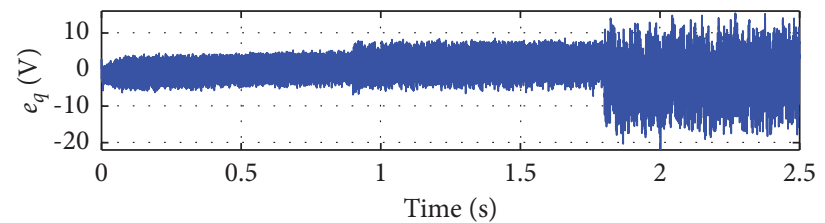

(b)

Figure 3: Tracking error surfaces under PI control strategy.

Thus, the derivative of $y_{d}$ can be approximately equal to the derivative of $y_{d}^{c}$. Figure 11 illustrates the estimation of lumped disturbance by disturbance-observer. Figure 12 compares the voltage control performance further, the real-time total harmonic distortion (THD) analysis of DER system output voltage under PI, backstepping, and DAFSC strategy. As can be seen, the proposed method ranks first on the current control performance with the least THD in all cases. 


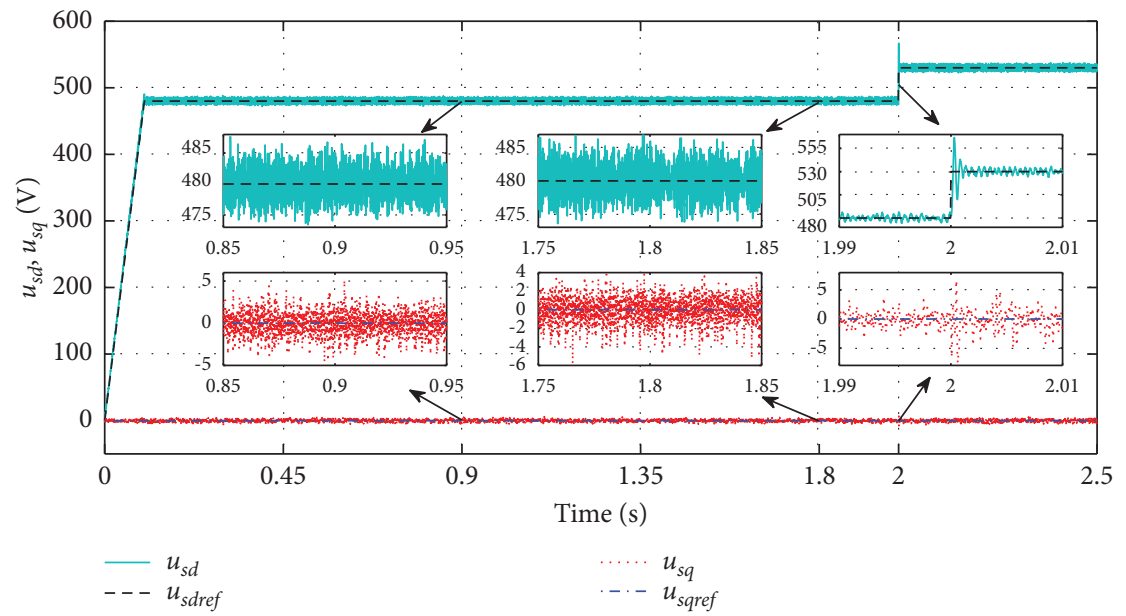

FIGURE 4: Voltage tracking effect under backstepping control strategy.

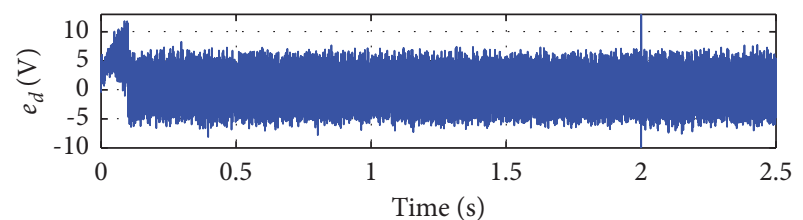

(a)

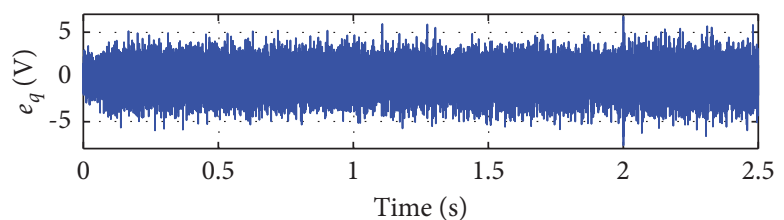

(b)

FIGURE 5: Tracking error surfaces under backstepping control strategy.

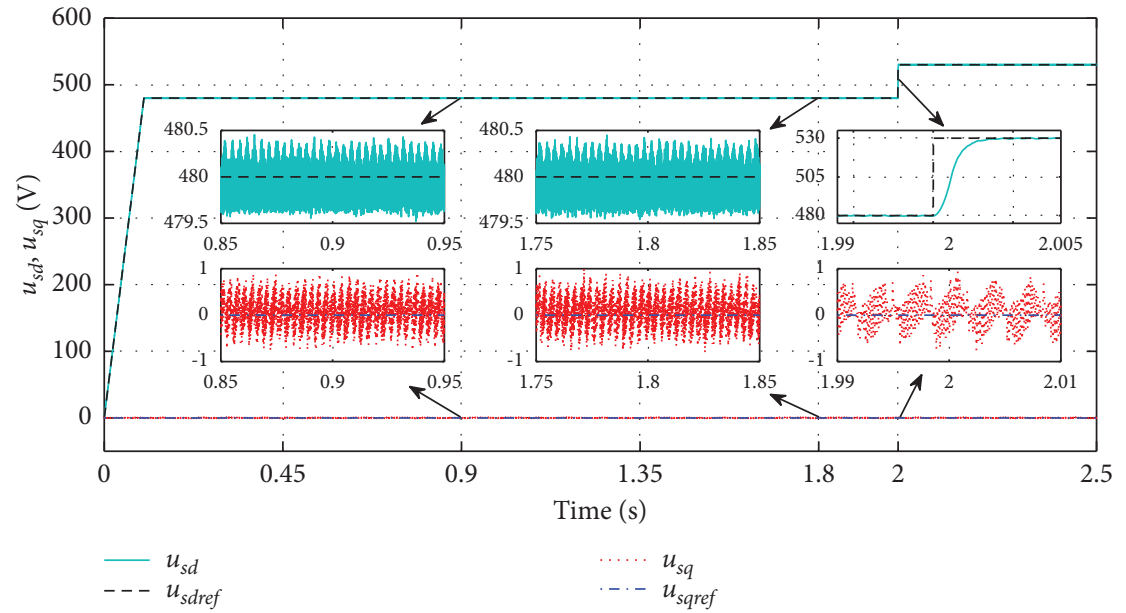

FIGURE 6: Voltage tracking effect under DAFSC strategy.

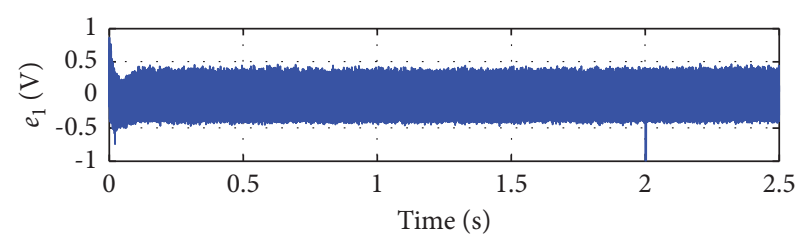

(a)

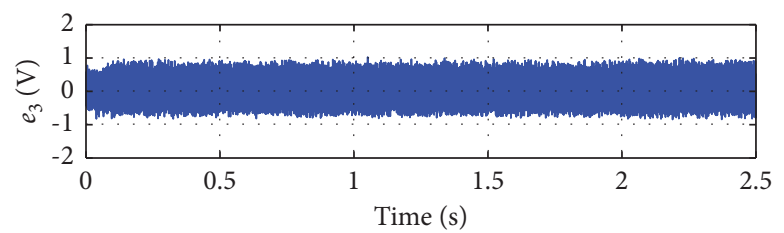

(b)

FIgURE 7: Tracking error surfaces under DAFSC strategy. 


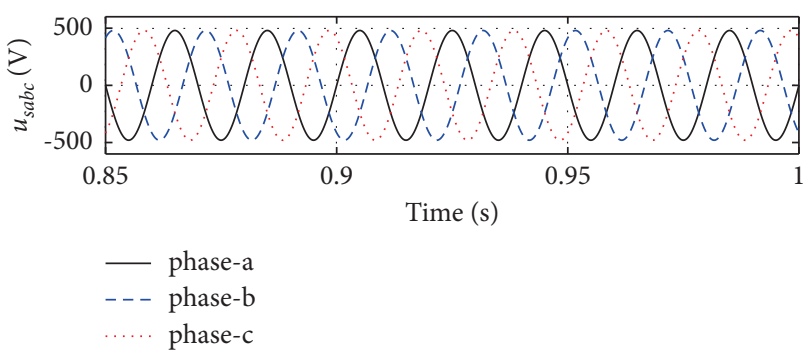

(a)

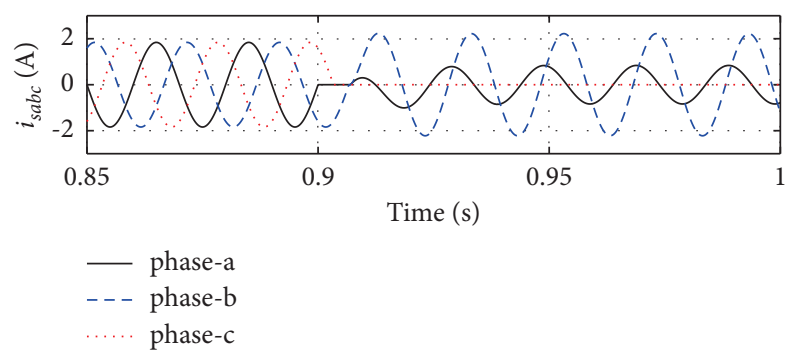

(b)

FIGURE 8: Waveforms of voltage and current under DAFSC strategy at $t=0.85 \mathrm{~s}-1 \mathrm{~s}$.

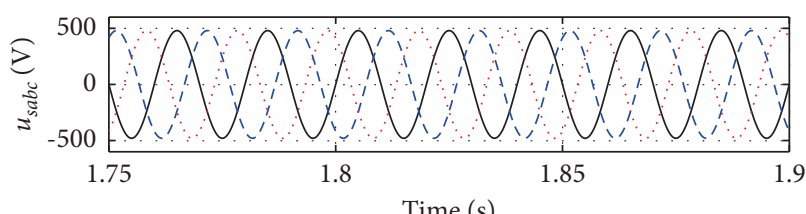

- phase-a

- - - phase-b phase-c

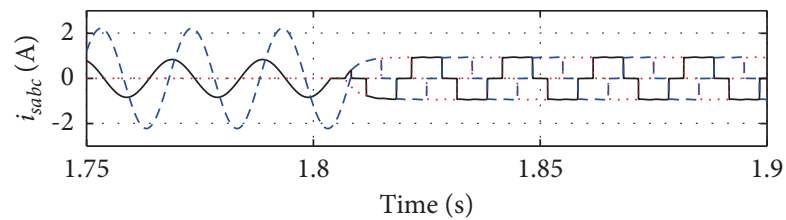

- phase-a

- - - phase-b

… phase-c

(a)

(b)

FIGURE 9: Waveforms of voltage and current under DAFSC strategy at $t=1.75 \mathrm{~s}-1.9 \mathrm{~s}$.

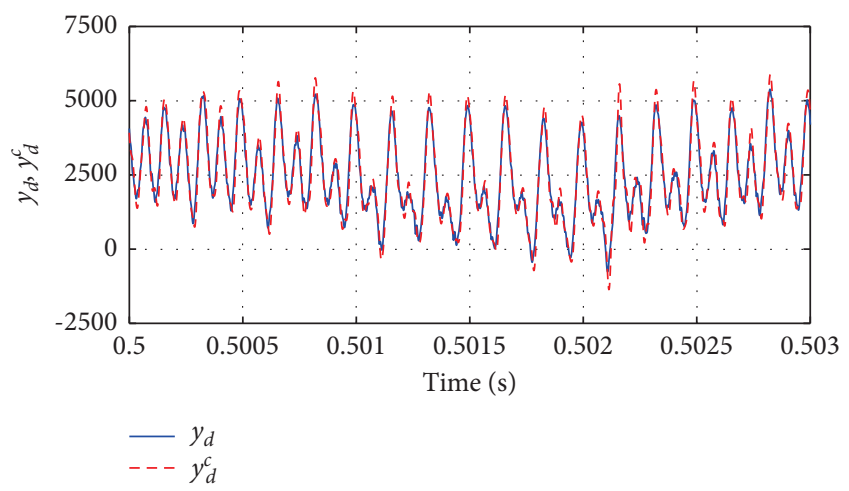

Figure 10: Input and output signal of SOSMD.

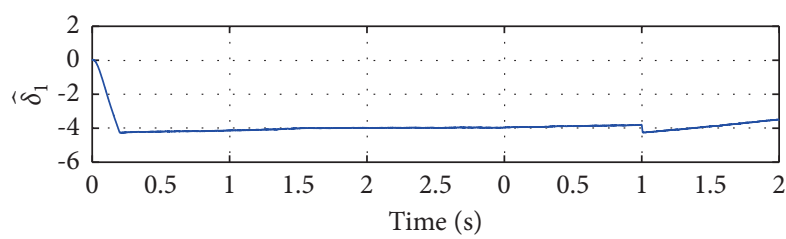

(a)

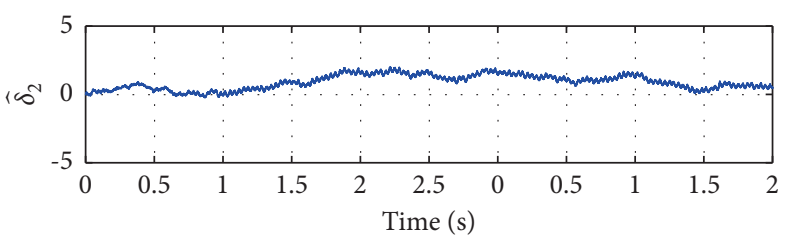

(b)

FiguRE 11: Estimation of lumped disturbance. 


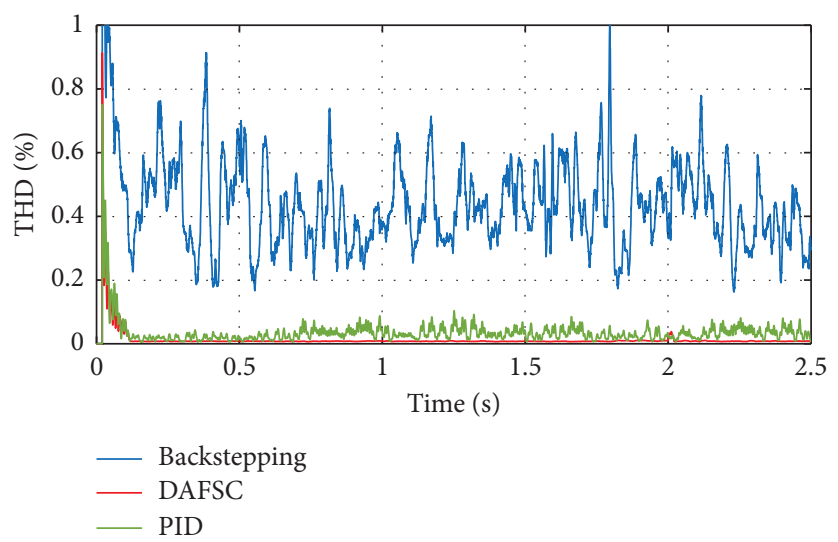

FIgURE 12: THD of DER system output voltage under different controllers.

\section{Conclusion}

This paper proposed a DAFSC method to deal with the voltage control problem for islanded DER systems, realizing the stable tracking of the system output voltage to the reference voltage no matter how the current is distorted. Meanwhile, the SOSMD is designed in the controller to solve the problem of calculation expansion in the backstepping control strategy. Then, the lumped disturbance of the system is observed by a designed disturbance-observer. Moreover, the nonlinear part of the dynamics model is approximated by FLS. The results indicate that the DAFSC strategy has better dynamic performance than the PI controller and backstepping controller, both on-load switching and reference voltage mutation. Moreover, the buffeting of the output voltage is kept within a small range by using the DAFSC controller. In the future work, our main research will be on how DER can effectively achieve better in the context of carbon neutrality and carbon peaks, and full utilization to achieve carbon emission reduction and related research on distributed energy storage systems.

\section{Data Availability}

The controller parameters used to support the findings of this study are included within the article.

\section{Conflicts of Interest}

The authors declare that they have no conflicts of interest.

\section{Acknowledgments}

This work was partially supported by the Open Research Fund of Jiangsu Collaborative Innovation Center for Smart Distribution Network (XTCX201902), Industry-UniversityResearch Cooperation Project of Jiangsu Province (BY2019023), Scientific Research Fund Project of Nanjing Institute of Technology (JCYJ201817), and China Scholarship Council (202106950045).

\section{References}

[1] Y. Chen, L. Hao, and G. Yin, "Distributed energy management of the hybrid AC/DC microgrid with high penetration of distributed energy resources based on ADMM," Mathematical Problems in Engineering, vol. 9, Article ID 1863855, 2021.

[2] C. Chandraratne, T. Naayagi Ramasamy, T. Logenthiran, and G. Panda, "Adaptive protection for microgrid with distributed energy resources," Electronics, vol. 9, no. 11, p. 1959, 2020.

[3] M. Pasetti, P. Ferrari, D. R. C. Silva, I. Silva, and E. Sisinni, "On the use of loRaWAN for the monitoring and control of distributed energy resources in a smart campus," Applied Sciences, vol. 10, no. 1, p. 320, 2020.

[4] R. Pause, I. D. van der Werf, and K. J. van den Berg, "Identification of pre-1950 synthetic organic pigments in artists' paints. A non-invasive approach using handheld Raman spectroscopy," Heritage, vol. 4, no. 3, pp. 1348-1365, 2021.

[5] Y. Fan, L. Fang, H. Wu et al., "A novel cascaded modular photovoltaic energy storage system for partial shading conditions," Applied Sciences, vol. 11, no. 12, p. 5552, 2021.

[6] W. Schinkel, T. van der Sande, and H. Nijmeijer, "State estimation for cooperative lateral vehicle following using vehicle-to-vehicle communication," Electronics, vol. 10, no. 6, p. 651, 2021.

[7] M. Yazdani-Damavandi, N. Neyestani, G. Chicco, M. ShafieKhah, and J. P. S. Catalao, "Aggregation of distributed energy resources under the concept of multienergy players in local energy systems," IEEE Transactions on Sustainable Energy, vol. 8, no. 4, pp. 1679-1693, 2017.

[8] K. E. Antoniadou-Plytaria, I. N. Kouveliotis-Lysikatos, P. S. Georgilakis, and N. D. Hatziargyriou, "Distributed and decentralized voltage control of smart distribution networks: models, methods, and future research," IEEE Transactions on Smart Grid, vol. 8, no. 6, pp. 2999-3008, 2017.

[9] Y. Dai, L. Zhang, G. Liu, C. Yang, D. Zhang, and X. Huang, "Prescribed-performance based finite-time adaptive fuzzy control for PV inverter in islanded systems," International Journal of Electrical Power \& Energy Systems, vol. 133, 2021.

[10] T.-V. Tran, T.-W. Chun, H.-H. Lee, H.-G. Kim, E.-C. Nho, and X. Huang, "PLL-based seamless transfer control between grid-connected and islanding modes in grid-connected inverters," IEEE Transactions on Power Electronics, vol. 29, no. 10, pp. 5218-5228, 2014. 
[11] E. Mayhorn, L. Xie, and K. Butler-Purry, "Multi-time scale coordination of distributed energy resources in isolated power systems," IEEE Transactions on Smart Grid, vol. 8, no. 2, pp. 998-1005, 2017.

[12] X. Zhou, J. Wang, and Y. Ma, "Linear active disturbance rejection control of grid-connected photovoltaic inverter based on deviation control principle," Energies, vol. 13, no. 15, p. 379, 2020.

[13] S. Gholami, M. Aldeen, and S. Saha, "Control strategy for dispatchable distributed energy resources in islanded microgrids," IEEE Transactions on Power Systems, vol. 33, no. 1, pp. 141-152, 2018.

[14] G. Lou, W. Gu, L. Wang, B. Xu, and M. Wu, W. Sheng, Decentralised secondary voltage and frequency control scheme for islanded microgrid based on adaptive state estimator," IET Generation, Transmission \& Distribution, vol. 11, no. 4, pp. 3683-3693, 2017.

[15] J. Chen and J. Chen, "Stability analysis and parameters optimization of islanded microgrid with both ideal and dynamic constant power loads," IEEE Transactions on Industrial Electronics, vol. 65, no. 4, pp. 3263-3274, 2021.

[16] M. B. Delghavi, S. Shoja-Majidabad, and A. Yazdani, "Fractional-order sliding-mode control of islanded distributed energy resource systems," IEEE Transactions on Sustainable Energy, vol. 7, no. 4, pp. 1482-1491, 2016.

[17] X. Wang and H. Wang, "Improved droop control strategy of multiple energy storage applications in an AC microgrid based on the state of charge," Electronics, vol. 10, no. 14, p. 1726, 2021.

[18] X. Yang, Y. Du, J. Su, L. Chang, Y. Shi, and J. Lai, “An optimal secondary voltage control strategy for an islanded multibus microgrid," IEEE Journal of Emerging and Selected Topics in Power Electronics, vol. 4, no. 4, pp. 1236-1246, 2016.

[19] A. Yazdani, "Control of an islanded distributed energy resource unit with load compensating feed-forward," in Proceedings of the IEEE Power and Energy Society General Meeting - Conversion and Delivery of Electrical Energy in the 21st Century, Pittsburgh, USA, July 2008.

[20] L. I. Minchala-Avila, L. Garza-Castanon, Y. Zhang, and H. J. A. Ferrer, "Optimal energy management for stable operation of an islanded microgrid," IEEE Transactions on Industrial Informatics, vol. 12, no. 4, pp. 1361-1370, 2016.

[21] M. M. A. Abdelaziz, H. E. Farag, and E. F. El-Saadany, "Optimum droop parameter settings of islanded microgrids with renewable energy resources," IEEE Transactions on Sustainable Energy, vol. 5, no. 2, pp. 434-445, 2014.

[22] S. M. Malik, X. Ai, Y. Sun, C. Zhengqi, and Z. Shupeng, "Voltage and frequency control strategies of hybrid AC/DC microgrid: a review," IET Generation, Transmission \& Distribution, vol. 11, no. 2, pp. 303-313, 2017.

[23] Z. Pan, K. Ezal, A. J. Krener, and P. V. Kokotovic, "Backstepping design with local optimality matching," IEEE Transactions on Automatic Control, vol. 46, no. 7, pp. 10141027, 2001.

[24] K. Ezal, Z. Zigang Pan, and P. V. Kokotovic, "Locally optimal and robust backstepping design," IEEE Transactions on Automatic Control, vol. 45, no. 2, pp. 260-271, 2000.

[25] Y. Shi, C. Shen, H. Fang, and H. Li, "Advanced control in marine mechatronic systems: a survey," IEEE, vol. 22, no. 3, pp. 1121-1131, 2017.

[26] J. A. Farrell, M. Polycarpou, M. Sharma, and W. Wenjie Dong, "Command filtered backstepping," IEEE Transactions on Automatic Control, vol. 54, no. 6, pp. 1391-1395, 2009.
[27] L. Huang, Y. Li, and S. Tong, "Command filter-based adaptive fuzzy backstepping control for a class of switched non-linear systems with input quantisation," IET Control Theory \& Applications, vol. 11, no. 12, pp. 1948-1958, 2017.

[28] W. Wenjie Dong, J. A. Farrell, and M. Polycarpou, V. Djapic, V. Djapic, and M. Sharma, Command filtered adaptive backstepping," IEEE Transactions on Control Systems Technology, vol. 20, no. 3, pp. 566-580, 2012.

[29] X. Wang, Y. Ma, J. Yu, L. Liu, and M. Li, "Fuzzy approximation-based adaptive command filtered control for induction motors," in Proceedings of the Chinese Control and Decision Conference (CCDC), Yinchuan, China, May 2016.

[30] C. Fu, L. Zhao, J. Yu, H. Yu, and C. Lin, "Neural networkbased command filtered control for induction motors with input saturation," IET Control Theory \& Applications, vol. 11, no. 5, pp. 2636-2642, 2017.

[31] J. Yu, P. Shi, W. Dong, and H. Yu, "Observer and commandfilter-based adaptive fuzzy output feedback control of uncertain nonlinear systems," IEEE Transactions on Industrial Electronics, vol. 62, no. 9, pp. 5962-5970, 2015.

[32] N. L. Díaz, J. G. Guarnizo, M. Mellado, J. C. Vasquez, and J. M. Guerrero, "A robot-soccer-coordination inspired control architecture applied to islanded microgrids," IEEE Transactions on Power Electronics, vol. 32, no. 4, pp. 27282742, 2017.

[33] Y. Dai, L. Zhang, G. Liu, D. Xu, and C. Yang, "Finite-time disturbance observer-based funnel voltage control strategy for vehicle-to-grid inverter in islanded mode," Proceedings of the Institution of Mechanical Engineers - Part I: Journal of Systems \& Control Engineering, vol. 235, no. 9, pp. 1571-1582, 2021.

[34] Y. Dai, G. Liu, C. Yang, D. Zhang, and X. Huang, "A novel no circulating current cooperative adaptive SoC balancing control for large-scale grid-connected electric vehicles," International Transactions on Electrical Energy Systems, vol. 30, no. 11, 2020.

[35] L. Zhao, B. Zhang, H. Yang, and Y. Wang, "Finite-time tracking control for pneumatic servo system via extended state observer," IET Control Theory \& Applications, vol. 11, no. 16, pp. 2808-2816, 2017.

[36] R. Rong-Jong Wai, J. Jing-Xiang Yao, and J. Jeng-Dao Lee, "Backstepping fuzzy-neural-network control design for hybrid maglev transportation system," IEEE Transactions on Neural Networks and Learning Systems, vol. 26, no. 2, pp. 302-317, 2015.

[37] Y. Dai, L. Zhang, D. Xu, Q. Chen, and X. Yan, "Anti-disturbance cooperative fuzzy tracking control of multi-PMSMs low-speed urban rail traction systems," IEEE Transactions on Transportation Electrification, 2021.

[38] X. Zhao, H. Yang, W. Xia, and X. Wang, "Adaptive fuzzy hierarchical sliding-mode control for a class of MIMO nonlinear time-delay systems with input saturation," IEEE Transactions on Fuzzy Systems, vol. 25, no. 5, pp. 1062-1077, 2017.

[39] J. Yu, P. Shi, and L. Zhao, "Finite-time command filtered backstepping control for a class of nonlinear systems," Automatica, vol. 92, pp. 173-180, 2018.

[40] Y. Dai, S. Ni, and D. Xu, "Disturbance-observer based prescribed-performance fuzzy sliding mode control for PMSM in electric vehicles," Engineering Applications of Artificial Intelligence, vol. 104, 2021. 